Rochester Institute of Technology

RIT Scholar Works

$12-2012$

GRM7 variants associated with age-related hearing loss based on auditory perception

Dina L. Newman

Laurel M. Fisher

Jeffrey Ohmen

Robert Parody

Chin-To Fong

See next page for additional authors

Follow this and additional works at: https://scholarworks.rit.edu/article 


\section{Authors}

Dina L. Newman, Laurel M. Fisher, Jeffrey Ohmen, Robert Parody, Chin-To Fong, Susan T. Frisina, Frances Mapes, David A. Eddins, Robert D. Frisina, and Rick A. Friedman 


\title{
GRM7 variants associated with age-related hearing loss based on auditory perception
}

\author{
Dina L. Newman a,f, ${ }^{*}$, Laurel M. Fisher ${ }^{b}$, Jeffrey Ohmen ${ }^{c}$, Robert Parody ${ }^{d}$, Chin-To Fong ${ }^{e}$, \\ Susan T. Frisina ${ }^{f}$, Frances Mapes ${ }^{f}$, David A. Eddins ${ }^{f, g}$, D. Robert Frisina ${ }^{f, g}$, Robert D. \\ Frisina $^{\mathrm{f}, \mathrm{g}}$, and Rick A. Friedman ${ }^{\mathrm{c},{ }^{* *}}$
}

Dina L. Newman: dina.newman@rit.edu; Laurel M. Fisher: LFisher@hei.org; Jeffrey Ohmen: JOhmen@hei.org; Robert Parody: rjpeqa@rit.edu; Chin-To Fong: Fong@urmc.rochester.edu; Susan T. Frisina: stfsusan@aol.com; Frances Mapes: fray.mapes@yahoo.com; David A. Eddins: deddins@usf.edu; D. Robert Frisina: frisina@usf.edu; Robert D. Frisina: rfrisina@usf.edu; Rick A. Friedman: RFriedman@hei.org

aThomas H. Gosnell School of Life Sciences, Rochester Institute of Technology, 153 Lomb Memorial Dr. Rochester, NY 14623, USA

bCenter for Clinical Studies, House Research Institute, Los Angeles, CA 90057, USA

${ }^{\circ}$ Cell Biology and Genetics Division, House Research Institute, Los Angeles, CA 90057, USA

dJohn D. Hromi Center for Quality and Applied Statistics, Rochester Institute of Technology, Rochester, NY 14623, USA

eDepartments of Pediatrics, Medical Humanities, Biochemistry and Biophysics, University of Rochester School of Medicine and Dentistry, Rochester, NY 14642, USA

fInternational Center for Hearing and Speech Research, National Technical Institute for the Deaf, Rochester Institute of Technology, Rochester, NY 14623, USA

9Global Center for Hearing \& Speech Research, Departments of Communication Sciences \& Disorders and Chemical \& Biomedical Engineering, University of South Florida, Tampa, FL 33620, USA

\section{Abstract}

Age-related hearing impairment (ARHI), or presbycusis, is a common condition of the elderly that results in significant communication difficulties in daily life. Clinically, it has been defined as a progressive loss of sensitivity to sound, starting at the high frequencies, inability to understand speech, lengthening of the minimum discernable temporal gap in sounds, and a decrease in the ability to filter out background noise. The causes of presbycusis are likely a combination of environmental and genetic factors. Previous research into the genetics of presbycusis has focused solely on hearing as measured by pure-tone thresholds. A few loci have been identified, based on a best ear pure-tone average phenotype, as having a likely role in susceptibility to this type of hearing loss; and GRM7 is the only gene that has achieved genome-wide significance. We examined the association of $G R M 7$ variants identified from the previous study, which used an European cohort with Z-scores based on pure-tone thresholds, in a European-American population from Rochester, NY $(N=687)$, and used novel phenotypes of presbycusis. In the present study mixed modeling analyses were used to explore the relationship of $G R M 7$ haplotype and SNP genotypes with various measures of auditory perception. Here we show that GRM7 alleles are associated primarily with peripheral measures of hearing loss, and particularly with speech detection in older adults. 


\section{Introduction}

Age-related hearing impairment (ARHI or presbycusis) is one of the top three chronic medical conditions of the aged, along with cardiovascular problems and arthritis (Dalstra et al., 2005; Parmet et al., 2007). The genetics of this complex trait are just beginning to be appreciated, with a significant interaction of genetic susceptibility and environmental components (Cruickshanks et al., 2010). Although most, if not all, people lose hearing acuity with age, empirical data have suggested that individuals have differing susceptibilities to ARHI. A strong genetic component to susceptibility is evidenced by its high heritability (approximately 50\%) in twin and family studies (Christensen et al., 2001; Gates et al., 1999; Karlsson et al., 1997; Wingfield et al., 2007). Environmental noise and ototoxic agents are well known to influence hearing in old age, and hormones also influence susceptibility (Guimaraes et al., 2006; Price et al., 2009; Tadros et al., 2005). Gender has long been established as a major factor in hearing loss with age; men's hearing tends to decline faster and earlier than women's (Dubno et al., 1997; Gates et al., 1999; Wiley et al., 2008). This is likely due to both environmental factors and the influence of sex hormones on the auditory system. For example, estrogen appears to be beneficial to preserving auditory function (Kilicdag et al., 2004; Kim et al., 2002).

Early studies addressing the genetic basis of ARHI in humans led to the identification of a voltage-gated potassium channel (KCNQ4, OMIM ID: 603537) (Van Eyken et al., 2006) and $\mathrm{N}$-acetyltransferase 2 (NAT2*6A, OMIM ID: 612182) (Unal et al., 2005; Van Eyken et al., 2007) as possible susceptibility genes. In one study, about 40 different candidate genes were tested based on their association with Mendelian forms of hearing loss; the most significant association was within an intron of the grainyhead-like 2 gene (GRHL2, OMIM ID: 608576) (Van Laer et al., 2008). Several linkage studies were attempted, but they either failed to identify any genome-wide significant peaks or exhibited such broad peaks that it was impossible to identify specific causative genes or mutations (DeStefano et al., 2003; Garringer et al., 2006; Huyghe et al., 2008).

A 500K-single nucleotide polymorphism (SNP) genome-wide association study of a large European cohort of older adults (Study 1) resulted in the identification of one haplotype within the glutamate metabotrophic receptor 7 (GRM7, OMIM ID: 604101) as harboring a significant risk allele for ARHI (Friedman et al., 2009). Friedman et al. also reported another, smaller, study (study 2), in which the locus was fine-mapped and confirmed in a second European cohort. Using two different genetic analysis methods, either a single intronic SNP or the adjacent haplotype block, showed the strongest signal in these two samples. GRM7 is thought to be central to maintaining glutamate synaptic transmission and homeostasis in the mammalian cochlea at the synapses between hair cells and the dendrites of afferent auditory nerve fibers. The presence of glutamate in excessive quantity has been suggested as a mechanism mediating neurotoxicity in auditory neurons (Pujol et al., 1990).

In the paper describing the original GRM7-ARHI association (Friedman et al., 2009), three genetic factors were described: a single SNP (rs11928865), and two haplotypes (Blocks 6 \& 7). SNPs are identified when the sequence of a single nucleotide (T, A, G, C) within a genome differs among individuals. This difference in DNA sequence may result in differences in the expression of proteins encoded by the gene, which may be related to a disease or other phenotypic aspect. Haplotype blocks are groups of SNPs that tend to be inherited together or "travel together" over successive generations. SNP rs11928865 falls within haplotype block 6, adjacent to haplotype block 7, and both haplotype blocks are found within intron 2 of the GRM7gene on chromosome 3. Given that there is no principled 
method by which SNP or haplotype information may be selected as the best indicator of the $G R M 7$ gene, we used both the haplotype and SNP definitions in the analyses.

No single measure captures the rich variety of hearing capacities in the auditory system. Clinically, pure-tone thresholds (PTs) are used to provide a "bird's eye view" of the basic level of hearing sensitivity (Fitzgibbons and Gordon-Salant, 1996; Frisina and Frisina, 1997; Schmiedt, 2010; Helfer and Vargo, 2009). Accurate measurement of pure-tone thresholds is dependent upon the alertness, cognitive and motoric abilities, and cooperation of the individual. Pure-tone thresholds are measured separately for each ear, in quiet, and at eight different frequencies (ranging from 0.25 to $8 \mathrm{kHz}$ ). The individual must push a button, raise a hand, or give verbal response to a tone in order to determine the quietest sound at which a frequency may be detected. The frequencies are chosen to test sounds in the speech range and, since the cochlea is tonotopically arranged, a sampling of frequencies will test the cochlea's function from base to apex. This clinical assessment of hearing results in eight measurements (each ear) or 16 data points for a single individual.

Pure-tone thresholds do not provide information about how well an individual can recognize speech or perceive speech (words or sentences). Further, the results of hearing tests conducted in quiet are often quite different than when that same test is conducted in noise. An individual who appears to have a normal audiogram (thresholds) may be impaired in a noisy situation, such as many older adults, who have difficulty understanding speech in the presence of background noise (Schneider et al., 2010). Hearing speech in noise performance may be more related to brainstem and auditory cortex functioning (Humes and Dubno, 2010), than strictly a product of end organ transduction. The complexity of measuring hearing increases with age, as discrete biological processes which combine to produce efficient speech processing may de-couple and, further, may have different trajectories over time (Birren and Fisher, 1991; Park and Reuter-Lorenz, 2009).

Given the complexity of measuring hearing and the clinical availability of pure-tone thresholds, the usual manner in which the aging phenotype has been defined has been to use pure-tone thresholds (e.g., ISO 7029; Acoustical Society of America, 1998). Methods to quantify the phenotype of ARHI have included a variety of statistical transformations of threshold data in order to reduce the dimensionality of the data set. Transformations of the data have included averaging across frequencies solely for the better ear, normalizing the average thresholds, principal components analysis, and Z-scores (age/sex-adjusted normally distributed data; Fransen et al., 2004). Each of these approaches defined ARHI using only derivations of PTs, despite the known complexity of the aging auditory system.

The ARHI phenotype associated with the GRM7 SNP rs11928865 (Friedman et al., 2009) was defined through using separate male and female $Z$-score transformations of the better ear's pure-tone average (PTA) at 2, 4, and $8 \mathrm{kHz}$, partialed for age. The $Z$-score, termed "high frequency" in the Friedman et al. paper, represented the distance from the median average threshold for males and females separately. Cases, or those subjects showing the effects of ARHI, were selected from both male and female distributions and showed a positive $Z$-score (poorer hearing than the median). Controls were selected from those showing a negative $Z$-score (better hearing than the median).

This methodology for selecting cases and controls is an effective approach when the underlying trait is more or less isomorphic with the measure used and the study design is a genome-wide association study. However, the Friedman et al. (2009) method separates older adults solely on their ability to hear high tones and does not address other aspects of the aging auditory system, such as aging effects on the other parts of the cochlea or speech understanding. The present data set and experimental design are uniquely positioned to 
provide supporting evidence for the association of $G R M 7$ genotypes with various measures of the aging auditory system more generally.

Age and gender effects can be modeled with genotype information in a battery of hearing measures, instead of using a single measure to create cases and controls. Several tests have been developed to assess human subjects for aspects of speech and temporal processing abilities that are localized to the central auditory system rather than just the cochlea. For example, the Hearing-in-Noise Test (HINT) is an assay of supra-threshold speech recognition in background noise, with components of spatial hearing abilities assessment (spatial release from masking). Supra-threshold gap detection tasks are also useful as measures of temporal features of the auditory system (Gordon-Salant and Fitzgibbons, 2001; Mazelová et al., 2003; Pichora-Fuller and Souza, 2003; Pichora-Fuller et al., 2007).

We chose mixed modeling techniques to appropriately analyze the dependencies inherent in multiple data points within PT data (Hox, 2010, Singer and Willett, 2003; West et al., 2007) and the hearing measures with data for each ear. Mixed modeling is an especially attractive technique for repeated measures designs and has moved from the domain of the specialist to a myriad of applications from medicine to sociology to economics (Hox, 2010). In essence, the dependencies in the repeated measures are modeled in the repeated level of analysis, leading to appropriate estimates of the fixed effects, here GRM7genotypes. The present investigation tested untransformed, or raw, PTs for both ears and measures related to speech perception to test for an association, or a statistically significant relationship, with GRM7 genotypes.

The data analysis proceeded in two steps in order to determine if an effect of GRM7 genotype could be detected on the hearing test results. At each step, we treated hearing variables as fully continuous variables and did not categorize a subset of subjects into cases or controls. The use of the hearing variables as continuous variables allowed an examination of the effects of GRM7 across a typical older adult population, whether the individual's hearing fell into a pre-defined category of showing ARHI or not.

First, analysis of covariance (ANCOVA) on "high frequency" (2, 4, $8 \mathrm{kHz})$ PTA for the better ear was used to examine the relationship of the single SNP and two haplotypes detected in the Friedman et al. (2009) work. The purpose of this step was to determine the effect of $G R M 7$ alleles, if any, in the present sample using different statistical methods, but keeping to the same underlying measurement (high PTs). The ANCOVA allows for the possibility of detecting an interaction between a GRM7 genotype, gender, and age at hearing test, on better ear PTA, something not possible with the previous design and analysis. This first analysis is not a replication of the Friedman et al. study, because we did not conduct the transformations as in the original paper, but supporting analyses to explicitly model relationships assumed in the previous work's data transformations and to gain a sense of the size of the genotype effect, if any. Second, mixed model analyses were carried out, beginning with more completely exploring the possible effects of $G R M 7$ genotype on all the pure-tone thresholds at the eight clinically assessed frequencies across each ear and including additional measures of hearing in the test battery.

The study had two objectives: (1) to determine the relationship, if any, between GRM7 haplotype or SNP allele and high frequency PTA in a well-described older adult sample, and (2) if a relationship holds, to test for a relationship in the additional hearing measures. If no relationship was found, then the basic work to determine the pathways in the auditory system impacted by $G R M 7$ would be of less interest. If a relationship was detected, then further work on the molecular pathways involving GRM7 in the auditory system would be indicated. 


\section{Material and methods}

\subsection{Study subjects}

The study cohort consisted of 687 individuals (59\% female) from the Rochester, NY greater metropolitan area, all of whom provided informed consent for their inclusion in the study. All subjects were white, over the age of 58 years (mean $71.3 \pm 7.3 \mathrm{y}$ ) and not related to any other subject in the study. Subjects donated a blood or buccal cell sample at the time of testing. Health and family histories were recorded. Individuals with the following conditions were excluded: conductive hearing loss (assessed by comparison of air conduction with bone conduction), severe head injury, labyrinthitis, mastoiditis, Meniere's disease, multiple sclerosis, neuropathy, dementia, stroke, history of seizures, hereditary hearing loss, ear infections, extensive noise exposure (self-report), and known ototoxic drug exposure.

\subsection{Audiological phenotype tests}

Volunteers from the Rochester cohort were paid $\$ 40$ for participation in a 4-h audiometric testing session, using protocols approved by both the Rochester Institute of Technology and University of Rochester Institutional Review Boards. The hearing tests included standard audiometric characterizations: pure-tone air conduction thresholds $(0.25,0.5,1,2,3,4,6,8$ $\mathrm{kHz})$ and bone conduction thresholds $(0.5,1,2,4 \mathrm{kHz})$, tympanometry to assess middle ear status/function, and speech reception thresholds (SRT). Additional testing included suprathreshold gap detection (a measure of temporal processing, presented at sound levels audible to all subjects) and HINT free-field speech-in-noise testing. The HINT results were analyzed using the release from masking (RFM) signal-to-noise ratio, thought to be a measure of binaural processing (Soli and Wong, 2008). These methods have been described in detail previously (Frisina et al., 2006; Guimaraes et al., 2006; Nilsson et al., 1994; Tadros et al., 2005). Table 1 shows the auditory test battery mean results in the Rochester cohort.

\subsection{Genotyping}

Genomic DNA was extracted from blood or buccal samples using the Gentra ${ }^{\circledR}$ PUREGENE ${ }^{\circledR}$ kit (now supplied by Qiagen). Genotyping of five GRM7SNPs (numbered in sequential order on the chromosome: GRM7-1 = rs11928865, GRM7-2 = rs9877154, GRM7-3 = rs3828472, GRM7-4 = rs9819783, GRM7-5 = rs11920109) was performed on DNA samples from all subjects using Pre-Designed TaqMan ${ }^{\circledR}$ SNP Assays from Applied Biosystems $^{\mathrm{TM}}$ with an Applied Biosystems ${ }^{\mathrm{TM}} 7500$ Real-Time PCR instrument under standard conditions. Random samples corresponding to each genotype were verified by direct sequencing using the BigDye ${ }^{\circledR}$ Terminator v3.1 Cycle Sequencing Kit from Applied Biosystems. All five SNPs were found to be in Hardy-Weinberg Equilibrium using the online "Hardy-Weinberg equilibrium calculator including analysis for ascertainment bias" (Rodriguez et al., 2009). Haplotypes were constructed from individual genotypes with the program PHASE (Stephens et al., 2001). Haplotype block 6 was constructed from rs11928865 and rs9877154, while haplotype block 7 was constructed from rs3828472, rs9819783 and rs11920109 (see Table 2). The allele frequencies of all five GRM7SNPs are shown in Table 2 and the haplotype frequencies are in Table 3. Since GRM7 block 7 haplotypes CTT, ATT, ACT and ACC counts made up 99\% of the sample, the other haplotypes were omitted from analyses.

\subsection{Association of genotype and phenotype: statistical methods}

"High frequency" PTA (HPTA) was calculated as a mean of the PTs at 2, 4, and $8 \mathrm{kHz}$. The speech intelligibility index (SII) was calculated using the PTs from each ear, using ANSI S3.5-1997 methodology (Acoustical Society of America, 1998). Not all subjects were able to detect the gap in noise, as evidenced by greatly lengthened detection responses (greater 
than $10 \mathrm{~s}$, see (Snell, 1997). The best gap detection time of the two repetitions at each frequency $(1$ and $4 \mathrm{kHz})$ was used in the analyses and any response greater than $10 \mathrm{~s}$ was coded as missing. In addition, not all subjects could complete the HINT test. Any noise composite score signal-to-noise ratio of greater than 0.0 (representing poor performance for normal hearers; Vermiglio, 2008) was coded as missing.

As noted above, the first step was to determine if the GRM7 genotypes significantly differed on HPTA for the better ear, with age at hearing test and gender as covariates. The interaction of genotype and gender was also added (full model). Second, the full model of fixed effects was estimated for the two haplotypes and the rs 11928865 allele for each hearing measure: PTs, SRT, SII, Gap Detection and HINT RFM ( $n=15$ separate analyses). The full or saturated model was estimated first using maximum likelihood (Harrell, 2010; Hox, 2010, Singer and Willett, 2003; West et al., 2007) and significant interactions were decomposed with univariate analyses. All analyses were conducted in SPSS ${ }^{\circledR} 19.0$, with statistical significance set at alpha $<0.05$.

The statistical hypothesis of interest is the test of a main effect of $G R M 7$ allele in each of the five dependent variables. Given that each dependent variable was examined for this main effect three times (one for each $G R M 7$ genotype), a Bonferroni correction was made to the alpha level for the main effect of $G R M 7$ allele. Thus, the significance level for the main effect of $G R M 7$ in each analysis was computed as $0.05 / 3=0.017$.

\section{Results}

The test sample included subjects with a wide range of hearing abilities, from those with excellent hearing for their age to those with severe hearing loss. On average, as shown in Fig. 1, females had better PT values than males and on average there was no strong advantage of one ear over the other; that is, most subjects had symmetrical hearing.

\subsection{GRM7 association with ARHI in the Rochester cohort}

3.1.1. High frequency pure-tone threshold average, better ear-An ANCOVA was conducted with each haplotype and rs11928865 genotype, with gender as a factor (male, female) and age as a continuous covariate. For each ANCOVA, age was a significant covariate (each $p<0.0001$ ) and gender was a significant main effect (each $p<0.0001$ ). Haplotype block 6 and 7 genotypes, and the rs11928865 genotype did not reach significance as a main effect (each $p>0.017$ ) or interaction with gender (each $p>0.017$ ). It appears that the best ear HPTA is not associated with GRM7.

3.1.2. Pure-tone threshold, both ears, eight frequencies-A saturated mixed model of pure-tone threshold by frequency and ear as repeated variables, with frequency, gender and age as fixed effects was conducted with each of the GRM7haplotype groups and the rs11928865 genotype. Three mixed model analyses were carried out.

Age, frequency, and gender were significant fixed effects (each $p<0.0001$ ) for all three $G R M 7$ genotype analyses. The gender by frequency interaction was significant (each $p<$ 0.0001 ) for all three $G R M 7$ genotype analyses. There were significant fixed effects of each GRM7 genotype (Haplotype 6, $p<0.0001$, Haplotype 7, $p<0.0001$, $\operatorname{rs11928865,} p<$ $0.015)$. Each of those p-values was less than the Bonferroni correction alpha value of 0.017 . The interaction between haplotype block 6 and gender was not significant $(p=0.05)$, but there was a significant interaction between haplo-type block 7 and gender $(p<0.0001$, see Fig. 2). There were no other significant effects. 
A mixed model of the PT by age and haplotype block 7 was conducted for males and females separately, in order to understand the interaction of haplotype block 7 and gender. There was a main effect of haplotype block 7 (each $p<0.0001$ ) for both the males and females, indicating that haplotype block 7 was significantly associated with PT within each gender. However, pairwise comparisons showed a complex relationship between genotype within gender, rendering the search for a risk allele within males and females difficult. Pairwise comparisons of the haplotype block 7 genotype within females showed that CTT/ CTT and CTT/ACT had significantly better hearing than ATT/ATT (each $p<0.0001$ ). Within the males, genotype CTT/CTT and CTT/ACT were among the five poorest hearing genotypes (each $p<0.001$ ). However, ATT/ATT genotype average threshold in the males was significantly poorer than the best hearing groups $(p<0.01)$, but not different from the poorest hearing groups. The data did not show any simple pattern of differences by gender.

3.1.3. Speech reception threshold, both ears-A saturated mixed model of SRT by ear as the repeated variable, with gender and age as fixed effects was conducted with each of the GRM7 haplotype blocks and the rs11928865 genotype. Three mixed model analyses were carried out. Age was significant across all three analyses $(p<0.001)$, but gender was not. The rs 11928865 genotype was not significant, haplotype blocks $6(p<0.021)$ and 7 ( $p<$ 0.013 ) were nominally significant effects, but only the haplo-type block 7 main effect was less than the Bonferroni corrected $p$-value 0.017 . No interaction was significant in any of the three analyses.

Pairwise comparisons among the haplotype block 6 genotypes showed that the TT/TT genotype tended to have the poorest average SRT relative to the other genotypes (see Fig. 3). Pairwise comparisons among the haplotype block 7 genotypes showed that CTT/CTT group tended to have the better SRT and ATT/ATT group tended to have the poorer SRT (see Fig. 3).

3.1.4. Speech intelligibility index (SII), both ears-A saturated mixed model of SII by ear as the repeated variable, with gender, PTA, and age as fixed effects was conducted with each of the GRM7haplotypes and the rs11928865 genotype. Pure-tone average (PTA) was included in this analysis to control for differences in hearing level influencing the SII. Three mixed model analyses were carried out.

Age, PTA, and gender were significant main effects in all three analyses (each $p<0.01$ ). There were no significant effects of the haplotype groups or rs11928865 genotype. There were no significant interactions in all three analyses.

3.1.5. Gap detection at $\mathbf{1}$ and $\mathbf{4} \mathbf{~ k H z}$-A saturated mixed model of Gap Detection by Frequency ( 1 and $4 \mathrm{kHz}$ ) as the repeated variable, with age as fixed effect was conducted with each of the GRM7 haplotype groups and the rs11928865 genotype. Three mixed model analyses were carried out. Age was a significant main effect in the analysis of haplotype block 6 and the rs 11928865 genotype (each $p<0.01$ ), but did not achieve significance in the analysis of haplotype block $7(p=0.053)$. There were no significant effects of the haplotype or single SNP genotype. There were no significant interactions in all three analyses.

3.1.6. HINT release from masking by side-A saturated mixed model of RFM by ear as the repeated variable, with age as fixed effect was conducted with each of the GRM7 haplotype blocks and the rs11928865 genotype. Three mixed model analyses were carried out. Age was a significant main effect for both haplotypes and the single SNP genotype (each $p<0.001$ ). Gender was a significant main effect across all three analyses (each $p<$ 0.004). There were no significant effects of the haplotype or single SNP genotype. There were no significant interactions in all three analyses. 


\section{Discussion}

The results from the Rochester subject population show that GRM7 is significantly associated with PT (individual thresholds across the ears) and SRT. Previous research had suggested a $G R M 7$ association with ARHI, as defined by $Z$-score better ear PTA (partialed for age and gender) in older adults. The present study has shown an association of GRM7 with SRT in older adults in addition to a comprehensive evaluation of PT. This is the first investigation of genetic associations with measures of speech detection, supporting the role of $G R M 7$ contributing to age-related hearing loss.

The present study is significant because it supports the finding of GRM7 association with ARHI (Friedman et al., 2009), and particularly with haplotype blocks 6 and 7. The $Z$-score used in the original study (Friedman et al., 2009) was similar to the HPTA phenotype used here; however, the $Z$-score method used age and gender in the calculation of the dependent value, whereas we used age and gender as covariates in the analysis. This difference in methodology likely contributes to our lack of a significant main effect of $G R M 7$ on the HPTA. In addition, the previous genome-wide study looked at the extreme ends of the PTA distribution, creating "cases and controls". In contrast, this study was able to take advantage of the entire sample, treating HPTA as a continuous variable.

Another GRM7 geno-phenotype study, on the Finnish Saami population, found association of a SNP downstream of $G R M 7$ with a principal component highly correlated with the $Z$ score of pure-tones (Van Laer et al., 2010). The presence of a different allele near the same gene in a historically isolated population is evidence of genetic heterogeneity as well as support for the importance of GRM7 in ARHI. Our procedure allowed for the explicit assessment of the respective contribution of age and gender to observed variability in auditory performance. The process of creating phenotype values based on age- and sexconditioned distributions presumes, without statistically testing that hypothesis, a significant relationship of age and gender to the hearing phenotype. This analytic process also does not allow for testing for the interaction effects of age and sex, the process presumes that the interaction estimate is zero. These are strict assumptions, which are not likely to hold in larger data sets, indeed, it was not supported in this data set. In contrast, we were able to test those associations explicitly. We discovered that the interaction of gender and genotype was significant for thresholds across frequencies and ear, suggesting that the pattern of GRM7 genotypes is different for women and men across age. This finding could not be detected using age- and sex-adjusted $Z$-scores of the previous investigations.

This study revealed that $G R M 7$ is not only associated with PT by frequency, but also SRT, a measure of hearing that has not been previously examined. The association of $G R M 7$ with SRT indicated a genetic association with aspects of speech detection. Speech detection deficits and PT elevations detract significantly from productivity, real-world quality of life, and psychological well-being for elderly people. Further research is indicated to understand the implications of the $G R M 7$ association with pure-tone thresholds and SRT.

Presbycusis is a highly prevalent communication problem for the majority of people over 65 years of age. Due to possible hormonal and gene/environment interactions, on average, older men are more severely affected than older women (Schmiedt, 2010), as was observed in the present subject sample (see Fig. 1). The percentage of the population affected by this ailment will increase in the near future even if there is no change in average lifespan. Given that the average lifespan may still increase, prevention of, slowing down the progression of, and/or treatment of ARHI comprise major scientific and translational medicine efforts. The ability to hear is frequently taken for granted until one suffers from hearing loss, and hearing losses in old age can prove to be a difficult adaptation process, resulting in communication 
difficulties at work and at home, leading to psychological problems and depression (Hogan et al., 2009). The findings of the present investigation are consistent with observations that genetics and environment interact in older individuals, linking age-related hearing loss to other neurodegenerative risk factors, including brain, cognitive and sensory functions declines (Lin, 2011; Lin et al., 2011).

Recent advances in the ability to assay hundreds of thousands of genetic variations in many individuals with high throughput techniques have allowed for the identification of susceptibility genes for specific diseases. Fundamental to these advances has been the discovery of reproducible quantitative traits. For many diseases, these measurements may have natural variation and fluctuations that increase the variability of the data obtained. ARHI is an ideal sensory deficit for these types of analyses due to the relatively stable, quantitative measurement of hearing loss, including determination of pure-tone and speech thresholds and supra-threshold hearing measures.

The human auditory system is a multi-component system that involves physical detection of the acoustic signal in the inner ear, processes to filter and amplify this signal, mechanisms to convert this signal into the code of the nervous system (action potentials), propagation of sound information to the brain, and then finally, interpretation by the regions of the central nervous system used for sound perception and sensory integration. Keeping the basic structure of the auditory system in mind, a primary goal is to derive a battery of maximally useful audiological tests and to use statistical analysis of the resulting data to quantitatively define hearing status for genetic analyses. Several decades ago, a research team headed by Schuknecht examined pure-tone audiometric data and suggested that ARHI was actually an umbrella diagnosis capturing up to four distinct etiologies within its diagnosis (Schuknecht, 1953, 1964, 1993; Schuknecht and Gacek, 1993). The usefulness of Schuknecht's approach to understanding the possible biological bases of presbycusis underlies the importance of utilizing a previously unprecedented, comprehensive battery of classic and experimental auditory tests for phenotypic characterization in the present investigation.

Any genetic analysis that solely uses threshold data to assess ARHI is bound to suffer from a reduction in power due to genetic heterogeneity. One approach to reducing this heterogeneity is to use broad test batteries of hearing ability to identify different sources of variability in data sets. Towards this goal, we collected a large cohort of aged individuals (average age $71 \mathrm{y}$ ), and then subjected them to an extensive battery of audiometric tests. These tests address different aspects or levels of the auditory system and can be used to identify groups of patients sharing similar phenotypic test results.

Finally, we showed that men and women have differing susceptibility to ARHI depending on their GRM7 genotypes. The mechanism of this gender interaction with PT is unknown, but it could be important in a clinical context. Future work investigating whether sex hormones directly or indirectly affect glutamate synaptic transmission in the cochlea is needed.

\section{Acknowledgments}

The authors wish to express their gratitude to all the volunteers who participated in the study. Gregory Warnes performed the calculation of SII; Karissa Raish, Dawn Mugrage and numerous undergraduate students at RIT assisted with DNA extraction and archiving of samples; and Elizabeth Hickman provided database support. This work was supported by grants from the National Institutes of Health (NIA: P01-AG009524 and K01-AG026394; NIDCD: R01-DC010215), the Seaver Foundation and the Schwartz Foundation (House Ear Institute). 


\section{Abbreviations}

$\begin{array}{ll}\text { ANCOVA } & \text { analysis of covariance } \\ \text { ANOVA } & \text { analysis of variance } \\ \text { ARHI } & \text { age-related hearing impairment } \\ \text { GRM7 } & \text { glutamate metabotropic receptor 7 gene } \\ \text { HINT } & \text { hearing in noise test } \\ \text { HPTA } & \text { high frequency pure tone average } \\ \text { PCR } & \text { polymerase chain reaction } \\ \text { PT } & \text { pure tone threshold (air conduction) } \\ \text { PTA } & \text { pure tone average } \\ \text { RFM } & \text { release from masking } \\ \text { SII } & \text { speech intelligibility index } \\ \text { SNP } & \text { single nucleotide polymorphism } \\ \text { SRT } & \text { speech reception threshold }\end{array}$

\section{References}

Acoustical Society of America. American National Standard: Methods for Calculation of the Speech Intelligibility Index. Acoustical Society of America; New York, NY: 1998. ANSI S3.5-1997

Birren, JE.; Fisher, LM. Aging and Slowing of Behavior: Consequences for Cognition and Survival, Nebraska Symposium on Motivation. Academic Press; New York, NY: 1991. p. 1-38.

Christensen K, Fredericksen H, Hoffman HJ. Genetic and environmental influences on self-reported reduced hearing in the old and oldest old. Journal of Geriatrics Society. 2001; 49:1512-1517.

Cruickshanks, KJ.; Zhan, W.; Zhong, W. Epidemiology of age-related hearing loss. In: Gordon-Salant, S.; Frisina, RD.; Popper, A.; Fay, RR., editors. The Aging Auditory System. Springer-Verlag; New York: 2010. p. 259-274.

Dalstra JA, Kunst AE, Borrell C, Breeze E, Cambois E, Costa G, Geurts JJ, Lahelma E, Van Oyen H, Rasmussen NK, Regidor E, Spadea T, Mackenbach JP. Socioeconomic differences in the prevalence of common chronic diseases: an overview of eight European countries. International Journal of Epidemiology. 2005; 34:316-326. [PubMed: 15737978]

DeStefano AL, Gates GA, Heard-Costa N, Myers RH, Baldwin CT. Genomewide linkage analysis to presbycusis in the Framingham Heart Study. Archives of Otolaryngology-Head \& Neck Surgery. 2003; 129:285-289. [PubMed: 12622536]

Dubno JR, Lee FS, Matthews LJ, Mills JH. Age-related and gender-related changes in monaural speech recognition. Journal of Speech, Language, and Hearing Research. 1997; 40:444-452.

Fitzgibbons PJ, Gordon-Salant S. Auditory temporal processing in elderly listeners. Journal of the American Academy of Audiology. 1996; 7:183-189. [PubMed: 8780991]

Fransen E, Van Laer L, Lemkens N, Caethoven G, Flothmann K, Govaerts P, Van de Heyning P, Van Camp G. A novel $Z$-score-based method to analyze candidate genes for age-related hearing impairment. Ear and Hearing. 2004; 25:133-141. [PubMed: 15064658]

Friedman R, Van Laer L, Huentelman M, Sheth S, Van Eyken E, Corneveaux J, Tembe W, Halperin R, Thorburn A, Thys S, Bonneux S, Fransen E, Huyghe J, Pyykkö I, Cremers C, Kremer H, Dhooge I, Stephens D, Orzan E, Pfister M, Bille M, Parving A, Sorri M, Van de Heyning P, Makmura L, Ohmen J, Linthicum FJ, Fayad J, Pearson J, Craig D, Stephan D, Van Camp G. GRM7 variants confer susceptibility to age-related hearing impairment. Human Molecular Genetics. 2009; 18:785-796. [PubMed: 19047183] 
Frisina D, Frisina R. Speech recognition in noise and presbycusis: relations to possible neural mechanisms. Hearing Research. 1997; 106:95-104. [PubMed: 9112109]

Frisina S, Mapes F, Kim S, Frisina D, Frisina R. Characterization of hearing loss in aged type II diabetics. Hearing Research. 2006; 211:103-113. [PubMed: 16309862]

Garringer HJ, Pankratz ND, Nichols WC, Reed T. Hearing impairment susceptibility in elderly men and the DFNA18 locus. Archives of Otolaryngology—Head \& Neck Surgery. 2006; 132:506-510. [PubMed: 16702566]

Gates G, Couropmitree N, Myers R. Genetic associations in age-related hearing thresholds. Archives of Otolaryngology—Head \& Neck Surgery. 1999; 125:654-659. [PubMed: 10367922]

Gordon-Salant S, Fitzgibbons P. Sources of age-related recognition difficulty for time-compressed speech. Journal of Speech, Language, and Hearing Research. 2001; 44:709-719.

Guimaraes P, Frisina S, Mapes F, Tadros S, Frisina D, Frisina R. Progestin negatively affects hearing in aged women. Proceedings of National Academy of Sciences USA. 2006; 103:14246-14249.

Harrell, FE. Regression Modeling Strategies: With Applications to Linear Models, Logistic Regression, and Survival Analysis. Spinger-Verlag; New York Inc., NY: 2010.

Helfer KS, Vargo M. Speech recognition and temporal processing in middle-aged women. Journal of the American Academy of Audiology. 2009; 20:264-271. [PubMed: 19927698]

Hogan A, O'Loughlin K, Miller P, Kendig H. The health impact of a hearing disability on older people in Australia. Journal of Aging and Health. 2009; 21:1098-1111. [PubMed: 19897778]

Hox, JJ. Multilevel Analysis: Techniques and Applications. 2. Routledge, NY: 2010.

Humes, LE.; Dubno, JR. Factors affecting speech understanding in older adults. In: Gordon-Salant, S.; Frisina, RD.; Popper, AN.; Fay, RR., editors. The Aging Auditory System. Springer, NY: 2010. p. 211-258.

Huyghe J, Van Laer L, Hendrickx J, Fransen E, Demeester K, Topsakal V, Kunst S, Manninen M, Jensen M, Bonaconsa A, Mazzoli M, Baur M, Hannula S, Mäki-Torkko E, Espeso A, Van Eyken E, Flaquer A, Becker C, Stephens D, Sorri M, Orzan E, Bille M, Parving A, Pyykkö I, Cremers C, Kremer H, Van de Heyning P, Wienker T, Nürnberg P, Pfister M, Van Camp G. Genome-wide SNP-based linkage scan identifies a locus on 8q24 for an age-related hearing impairment trait. American Journal of Human Genetics. 2008; 83:401-407. [PubMed: 18760390]

Karlsson K, Harris J, Svartengren M. Description and primary results from an audiometric study of male twins. Ear and Hearing. 1997; 18:114-120. [PubMed: 9099560]

Kilicdag EB, Yavuz H, Bagis T, Tarim E, Erkan AN, Kazanci F. Effects of estrogen therapy on hearing in postmenopausal women. American Journal of Obstetrics and Gynecology. 2004; 190:77-82. [PubMed: 14749639]

Kim SH, Kang BM, Chae HD, Kim CH. The association between serum estradiol level and hearing sensitivity in postmenopausal women. Obstetrics \& Gynecology. 2002; 99:726-730. [PubMed: 11978279]

Lin FR. Hearing loss and cognition among older adults in the United States. The Journals of Gerontology Series A: Biological Sciences and Medical Sciences. 2011; 66A:1131-1136.

Lin FR, Ferrucci L, Metter EJ, An Y, Zonderman AB, Resnick SM. Hearing loss and cognition in the Baltimore longitudinal study of aging. Neuropsychology. 2011; 25:763-770. [PubMed: 21728425]

Mazelová J, Popelar J, Syka J. Auditory function in presbycusis: peripheral vs. central changes. Experimental Gerontology. 2003; 38:87-94. [PubMed: 12543265]

Nilsson M, Soli SD, Sullivan JA. Development of the Hearing in Noise Test for the measurement of speech reception thresholds in quiet and in noise. Journal of the Acoustical Society of America. 1994; 95:1085-1099. [PubMed: 8132902]

Park DC, Reuter-Lorenz P. The adaptive brain: aging and neurocognitive scaffolding. The Annual Review of Psychology. 2009; 60:173-196.

Parmet S, Lynm C, Glass RM. JAMA patient page. Adult hearing loss. JAMA. 2007; 298:130. [PubMed: 17609496]

Pichora-Fuller M, Souza P. Effects of aging on auditory processing of speech. International Journal of Audiology. 2003; 42 (Suppl 2):S11-S16. 
Pichora-Fuller M, Schneider B, Macdonald E, Pass H, Brown S. Temporal jitter disrupts speech intelligibility: a simulation of auditory aging. Hearing Research. 2007; 223:114-121. [PubMed: 17157462]

Price K, Zhu X, Guimaraes P, Vasilyeva O, Frisina R. Hormone replacement therapy diminishes hearing in peri-menopausal mice. Hearing Research. 2009; 252:29-36. [PubMed: 19269311]

Pujol R, Rebillard G, Puel J, Lenoir M, Eybalin M, Recasens M. Glutamate neurotoxicity in the cochlea: a possible consequence of ischaemic or anoxic conditions occurring in ageing. Acta OtoLaryngologica Supplementum. 1990; 476:32-36. [PubMed: 1982389]

Rodriguez S, Gaunt TR, Day IN. Hardy-Weinberg equilibrium testing of biological ascertainment for Mendelian randomization studies. American Journal of Epidemiology. 2009; 169:505-514. [PubMed: 19126586]

Schmiedt, RA. The physiology of cochlear presbycusis. In: Gordon-Salant, S.; Frisina, RD.; Popper, AN.; Fay, RR., editors. The Aging Auditory System. Springer, NY: 2010. p. 31-33.p. 18

Schneider, BA.; Pichora-Fuller, K.; Daneman, M. Effects of senescent changes in audition and cognition on spoken language comprehension. In: Gordon-Salant, S.; Frisina, RD.; Popper, AN.; Fay, RR., editors. The Aging Auditory System. Springer; NY: 2010. p. 167-210.

Schuknecht H. Lesions of the organ of Corti. Transactions of the American Academy of Ophthalmology \& Otolaryngology. 1953; 57:366-383.

Schuknecht H. Further observations on the pathology of presbycusis. Archives of OtolaryngologyHead \& Neck Surgery. 1964; 80:369-382.

Schuknecht, HF. Pathology of The Ear. 2. Lea and Febiger; Philadelphia: 1993.

Schuknecht H, Gacek M. Cochlear pathology in presbycusis. Annals of Otology, Rhinology and Laryngology. 1993; 102:1-16.

Singer, JD.; Willett, JB. Modeling Change and Event Occurrence. Oxford University Press; NY: 2003. Applied Longitudinal Data Analysis.

Snell KB. Age-related changes in temporal gap detection. Journal of the Acoustical Society of America. 1997; 101:2214-2220. [PubMed: 9104023]

Soli SD, Wong LL. Assessment of speech intelligibility in noise with the Hearing in Noise Test. International Journal of Audiology. 2008; 47:356-361. [PubMed: 18569108]

Stephens M, Smith NJ, Donnelly P. A new statistical method for haplotype reconstruction from population data. American Journal of Human Genetics. 2001; 68:978-989. [PubMed: 11254454]

Tadros S, Frisina S, Mapes F, Frisina D, Frisina R. Higher serum aldosterone correlates with lower hearing thresholds: a possible protective hormone against presbycusis. Hearing Research. 2005; 209:10-18. [PubMed: 16039078]

Unal M, Tamer L, Doğruer Z, Yildirim H, Vayisoğlu Y, Camdeviren H. N-acetyltransferase 2 gene polymorphism and presbycusis. Laryngoscope. 2005; 115:2238-2241. [PubMed: 16369173]

Van Eyken E, Van Laer L, Fransen E, Topsakal V, Lemkens N, Laureys W, Nelissen N, Vandevelde A, Wienker T, Van De Heyning P, Van Camp G. KCNQ4: a gene for age-related hearing impairment? Human Mutation. 2006; 27:1007-1016. [PubMed: 16917933]

Van Eyken E, Van Camp G, Fransen E, Topsakal V, Hendrickx J, Demeester K, Van de Heyning P, Mäki-Torkko E, Hannula S, Sorri M, Jensen M, Parving A, Bille M, Baur M, Pfister M, Bonaconsa A, Mazzoli M, Orzan E, Espeso A, Stephens D, Verbruggen K, Huyghe J, Dhooge I, Huygen P, Kremer H, Cremers C, Kunst S, Manninen M, Pyykkö I, Lacava A, Steffens M, Wienker T, Van Laer L. Contribution of the N-acetyl-transferase 2 polymorphism NAT2*6A to age-related hearing impairment. Journal of Medical Genetics. 2007; 44:570-578. [PubMed: 17513527]

Van Laer L, Huyghe JR, Hannula S, Van Eyken E, Stephan DA, Maki-Torkko E, Aikio P, Fransen E, Lysholm-Bernacchi A, Sorri M, Huentelman MJ, Van Camp G. A genome-wide association study for age-related hearing impairment in the Saami. European Journal of Human Genetics. 2010; 18:685-693. [PubMed: 20068591]

Van Laer L, Van Eyken E, Fransen E, Huyghe J, Topsakal V, Hendrickx J, Hannula S, Mäki-Torkko E, Jensen M, Demeester K, Baur M, Bonaconsa A, Mazzoli M, Espeso A, Verbruggen K, Huyghe J, Huygen P, Kunst S, Manninen M, Konings A, Diaz-Lacava A, Steffens M, Wienker T, Pyykkö I, Cremers C, Kremer H, Dhooge I, Stephens D, Orzan E, Pfister M, Bille M, Parving A, Sorri M, 
Van de Heyning P, Van Camp G. The grainyhead like 2 gene (GRHL2), alias TFCP2L3, is associated with age-related hearing impairment. Human Molecular Genetics. 2008; 17:159-169. [PubMed: 17921507]

Vermiglio AJ. The American English hearing in noise test. International Journal of Audiology. 2008; 47:386-387. [PubMed: 18569120]

West, BT.; Welch, KB.; Galecki, AT. Linear Mixed Models: A Practical Guide Using Statistical Software. Chapman \& Hall/CRC; NY: 2007.

Wiley TL, Chappell R, Carmichael L, Nondahl DM, Cruickshanks KJ. Changes in hearing thresholds over 10 years in older adults. Journal of the American Academy of Audiology. 2008; 19:281-292. quiz 371. [PubMed: 18795468]

Wingfield A, Panizzon M, Grant M, Toomey R, Kremen W, Franz C, Jacobson K, Eisen S, Lyons M. A twin-study of genetic contributions to hearing acuity in late middle age. The Journals of Gerontology Series A: Biological Sciences and Medical Sciences. 2007; 62:1294-1299. 


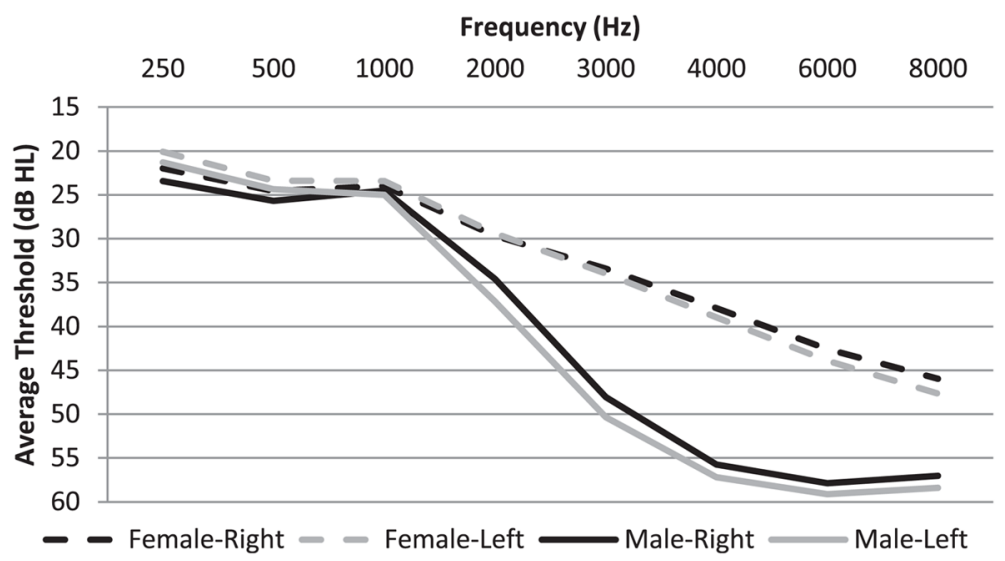

Fig. 1.

Pure-tone thresholds by test frequency and gender for all subjects $(n=687)$. 


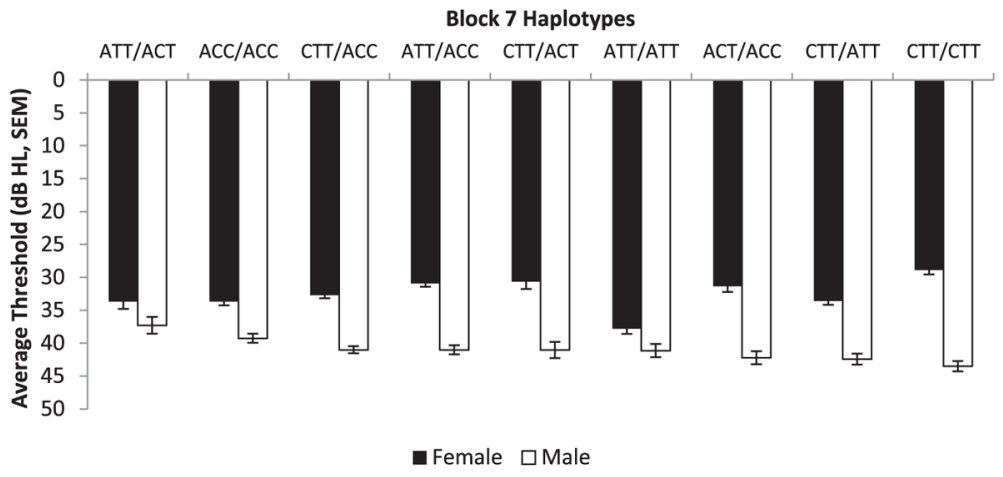

Fig. 2.

Average pure-tone threshold by gender and haplotype block 7 (standard error of the mean). The haplotypes are ordered by average threshold for the males. Note the genotype CTT/ CTT: the females had the best hearing but the males had the poorest hearing. 

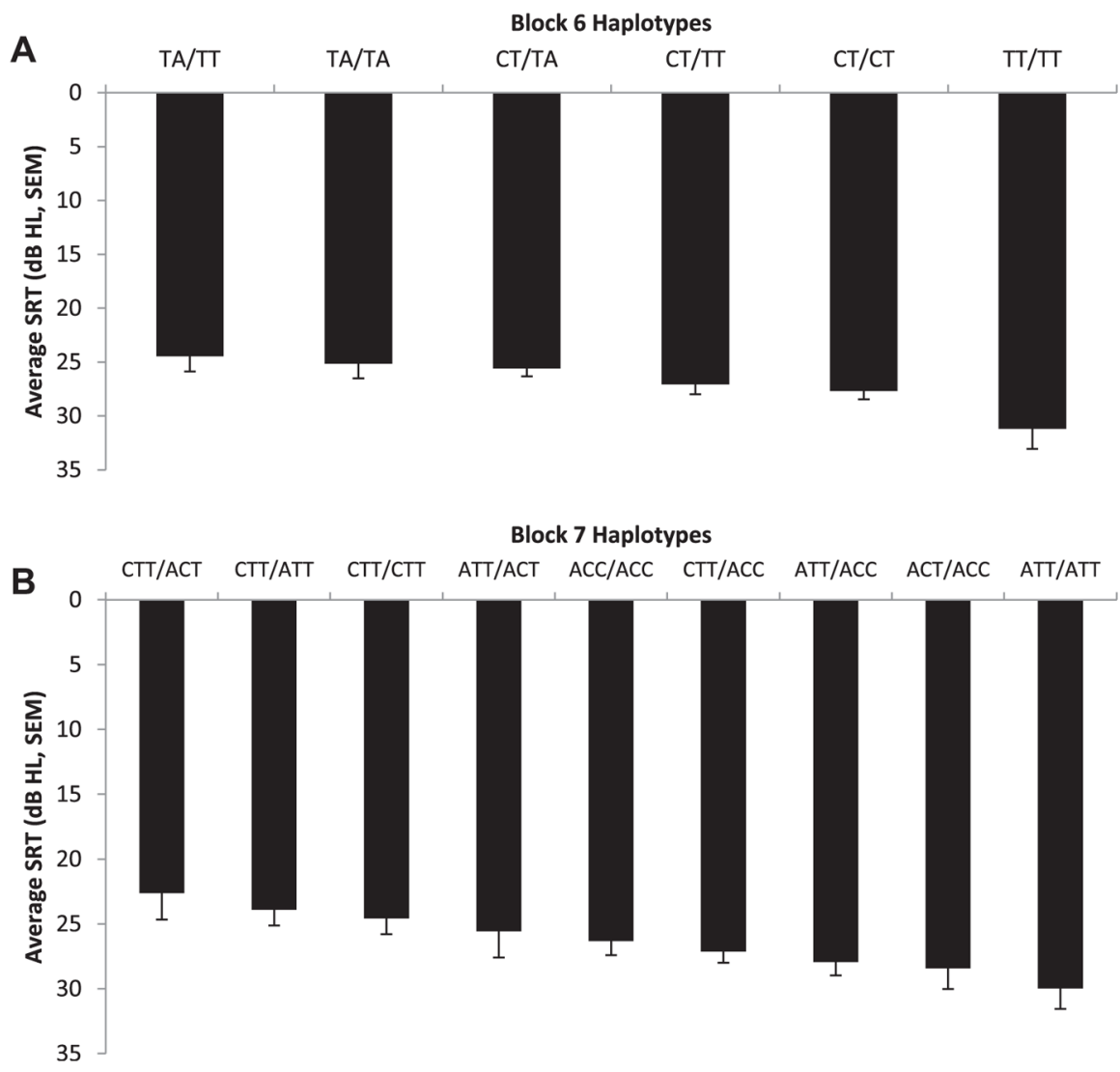

Fig. 3.

Average speech reception threshold (dB, standard error of the mean) by haplotype block 6 . Groups are ordered by average. 


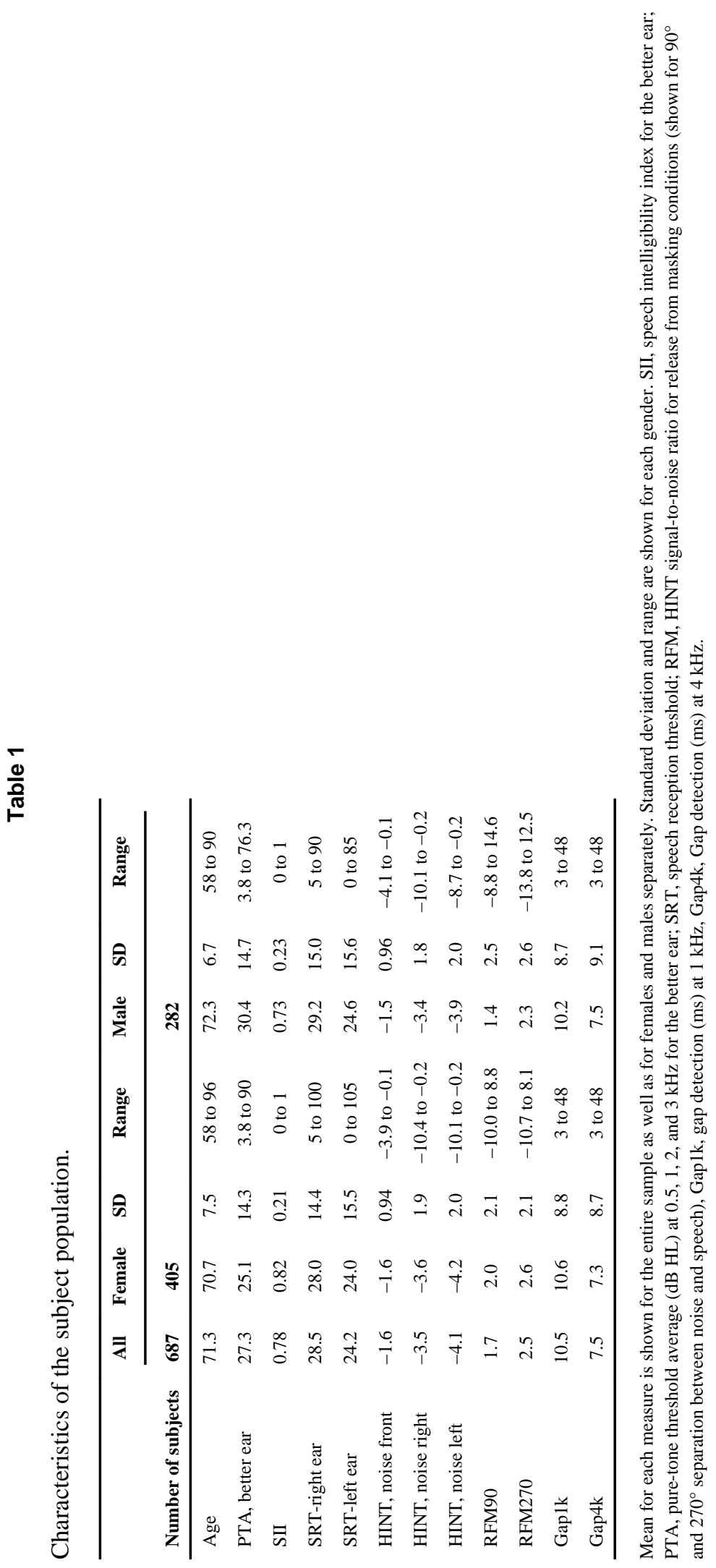

Hear Res. Author manuscript; available in PMC 2013 December 01. 
Table 2

Allele frequencies of $G R M 7$ SNPs.

\begin{tabular}{llll}
\hline SNP ID number & Alleles $(\mathbf{p}, \mathbf{q})^{\boldsymbol{a}}$ & Minor allele frequency & Haplotype block \\
\hline rs11928865 & C, T & 0.48 & 6 \\
rs9877154 & T, A & 0.28 & 6 \\
rs3828472 & A, C & 0.31 & 7 \\
rs9819783 & T, C & 0.44 & 7 \\
rs11920109 & T, C & 0.37 & 7 \\
\hline
\end{tabular}

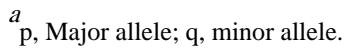


